\title{
Structure and phase transitions of monolayers of intermediate-length $n$-alkanes on graphite studied by neutron diffraction and molecular dynamics simulation
}

\author{
A. Diama, ${ }^{\text {a) }}$ B. Matthies, ${ }^{\text {b) }}$ K. W. Herwig,${ }^{\text {c) }}$ F. Y. Hansen, ${ }^{\text {d) }}$ L. Criswell, H. Mo, ${ }^{e)}$ M. Bai, and \\ H. Taub ${ }^{\mathrm{f}}$ \\ Department of Physics and Astronomy and the University of Missouri Research Reactor, \\ University of Missouri, Columbia, Missouri 65211, USA
}

(Received 3 June 2009; accepted 3 August 2009; published online 25 August 2009)

\begin{abstract}
We present evidence from neutron diffraction measurements and molecular dynamics (MD) simulations of three different monolayer phases of the intermediate-length alkanes tetracosane $\left(n-\mathrm{C}_{24} \mathrm{H}_{50}\right.$ denoted as $\left.\mathrm{C} 24\right)$ and dotriacontane $\left(n-\mathrm{C}_{32} \mathrm{H}_{66}\right.$ denoted as $\left.\mathrm{C} 32\right)$ adsorbed on a graphite basal-plane surface. Our measurements indicate that the two monolayer films differ principally in the transition temperatures between phases. At the lowest temperatures, both C24 and C32 form a crystalline monolayer phase with a rectangular-centered (RC) structure. The two sublattices of the $\mathrm{RC}$ structure each consists of parallel rows of molecules in their all-trans conformation aligned with their long axis parallel to the surface and forming so-called lamellas of width approximately equal to the all-trans length of the molecule. The RC structure is uniaxially commensurate with the graphite surface in its [110] direction such that the distance between molecular rows in a lamella is $4.26 \AA=\sqrt{3} a_{g}$, where $a_{g}=2.46 \AA$ is the lattice constant of the graphite basal plane. Molecules in adjacent rows of a lamella alternate in orientation between the carbon skeletal plane being parallel and perpendicular to the graphite surface. Upon heating, the crystalline monolayers transform to a "smectic" phase in which the inter-row spacing within a lamella expands by $\sim 10 \%$ and the molecules are predominantly oriented with the carbon skeletal plane parallel to the graphite surface. In the smectic phase, the MD simulations show evidence of broadening of the lamella boundaries as a result of molecules diffusing parallel to their long axis. At still higher temperatures, they indicate that the introduction of gauche defects into the alkane chains drives a melting transition to a monolayer fluid phase as reported previously. (c) 2009 American Institute of Physics.
\end{abstract}

[DOI: $10.1063 / 1.3212095]$

\section{INTRODUCTION}

Films of intermediate-length alkane molecules $\left(\mathrm{C}_{n} \mathrm{H}_{2 n+2}\right.$; $15<n<40)$ are of interest as prototypical systems for understanding the interfacial behavior of more complex polymers. These chainlike molecules are large and flexible enough that their conformational changes can significantly affect the translational order within a monolayer film; and yet they are small enough to be tractable in detailed molecular dynamics (MD) simulations.

From a fundamental standpoint, it is of interest to investigate how the monolayer structure of intermediate-length alkanes may depend on the molecular conformation. Such structural characterization may aid in understanding the mi-

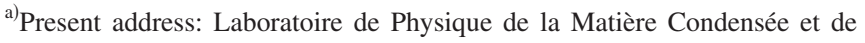
Technologie (LPMCT), Université de Cocody, Abidjan, Côte d'Ivoire.

${ }^{b}$ Present address: The Goodyear Tire and Rubber Company, Corporate Research Division, 142 Goodyear Boulevard, Akron, OH 44305.

${ }^{c}$ Present address: Neutron Scattering Science Division, Oak Ridge National Laboratory, Oak Ridge, TN 37830.

${ }^{d)}$ Permanent address: Department of Chemistry, Technical University of Denmark, IK 207 DTU, DK-2800 Lyngby, Denmark.

e) Present address: Advanced Optowave Corporation, 1170 Lincoln Ave., Unit No. 10, Holbrook, New York 11741.

${ }^{\mathrm{f})}$ Electronic mail: taubh@missouri.edu.
}

croscopic mechanism by which a flexible-chain molecule adsorbs on a surface ${ }^{1}$ and desorbs upon heating. ${ }^{2}$ It may also help explain whether or not complete wetting of the solid film occurs at a higher coverage. ${ }^{3}$ Knowledge of the monolayer structure is also essential for understanding its dynamical properties, including both vibratory ${ }^{4}$ and diffusive $e^{5,6}$ motion.

Intermediate-length alkanes are also of considerable technological interest as the principal constituents of commercial lubricants. Progress in understanding their monolayer structure and dynamics could potentially impact technologically important issues such as evaporation of thin lubricant films from the surfaces of magnetic storage disks and the lubricating characteristics of alkane films.

Almost 40 years ago, Groszek ${ }^{7}$ proposed a structural model for $n$-alkane monolayers adsorbed on the graphite basal-plane surface based on his adsorption isotherm measurements. He noted that it would be energetically favorable for a single $n$-alkane molecule in an all-trans configuration to adsorb with its carbon skeletal plane parallel to the graphite surface and with an outer plane of hydrogen atoms occupying the centers of the graphite carbon hexagons, as shown in Fig. 1(a). Using this model, he was able to estimate the area occupied by the alkane molecule and to argue why the 


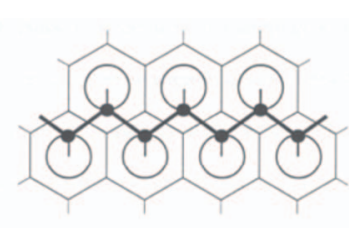

(a)

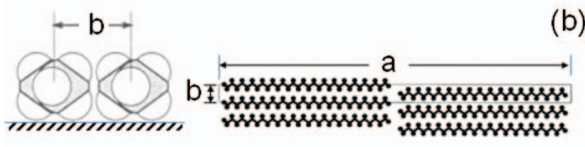

(b)

$-b \rightarrow$

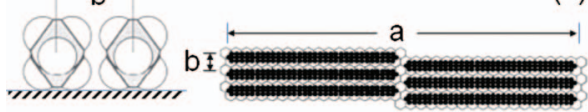

$\frac{b}{2} \rightarrow$

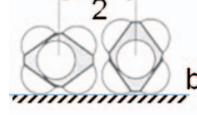

$b$ Hssssssssss mssssssses

FIG. 1. (a) Groszek's model of an all-trans alkane molecule adsorbed on a graphite basal-plane surface (from Ref. 7). Open circles represent hydrogen atoms located nearest to the graphite surface and solid circles represent carbon atoms in the alkane molecules. (b) Model of a low-density incommensurate monolayer phase of an intermediate-length alkane molecule. The molecules are oriented with the plane of the carbon backbone parallel to the graphite surface but are not in registry with the graphite surface as in (a). (c) Model of an alkane monolayer commensurate with the graphite surface in the b-direction and in which the plane of the carbon backbone is perpendicular to the graphite surface (see Refs. 12 and 13). (d) Model of a highdensity alkane monolayer phase that is commensurate with graphite surface in the b-direction, but in which the plane of the alkane carbon backbone alternates between being parallel and perpendicular to the graphite surface (from Ref. 16). In (b)-(d), the left panels show an end view looking along the long axis of the molecule. The right panels depict a top view of the monolayer.

longer alkane molecule would adsorb preferentially from a heptane solution.

Previous experimental studies of the monolayer structure of intermediate-length alkanes have focused on high-quality solid surfaces of graphite, ${ }^{7-21} \mathrm{Ag}(111),{ }^{22,23}$ and $\mathrm{SiO}_{2} \cdot{ }^{3,24,25}$ Of these systems, probably the best characterized are monolayers of C32 and C24 on high-quality graphite basal-plane surfaces that have been investigated by scanning tunneling microscopy (STM), ${ }^{10-14}$ x-ray diffraction, ${ }^{15}$ and neutron scattering. ${ }^{16-21}$ Graphite substrates also have the advantage that relatively reliable empirical atom-atom potentials are available for representing the molecule/graphite basal-plane interaction.

Neutron diffraction measurements on C24 and C32 adsorbed on graphite basal-plane surfaces ${ }^{16-19,21}$ have found two different solid monolayer structures at room temperature, depending on whether the film is deposited from its vapor phase or from a solution with heptane. Both structures appear to be thermodynamically stable and share a rectangular-centered (RC) unit cell containing two molecules oriented with their long axis parallel to the graphite surface. The long side of the unit cell $a$ is approximately equal to twice the all-trans length of the molecule, $87 \pm 1.5$ and $65.0 \pm 1.5 \AA$ for C32 and C24, respectively.
However, these two monolayer structures found for both C24 and C32 differ in several respects. ${ }^{16}$ In the case of the vapor-deposited monolayer, the molecules orient with their carbon skeletal plane parallel to the graphite surface, as shown in Fig. 1(b) with $b$ lattice constant in the range of 4.53-4.66 $\AA$, depending on coverage. In contrast, when the C24 or C32 monolayer is deposited from solution or when at least a monolayer of heptane is adsorbed above the vapordeposited phase, the longer alkane monolayer contracts uniaxially in the b-direction. The skeletal plane of the molecules now alternates in this direction from parallel to perpendicular to the graphite surface, as shown in Fig. 1(d), resulting in a doubling of the unit cell. ${ }^{16}$ This structure is partially commensurate with the graphite surface with $b$ $=8.52 \AA=2 \sqrt{3} a_{g}$, where $a_{g}=2.46 \AA$ is the lattice constant of the graphite basal plane. As discussed in Ref. 16, neutron diffraction measurements are inconsistent with the model in Fig. 1(c) in which all molecules are oriented with their carbon skeletal plane perpendicular to the surface.

In addition, the vapor-deposited C24 and C32 monolayers have a shorter coherence length $L_{b}$ (typically $\sim 40 \AA$ ) along the b-direction compared to a value of $L_{b} \sim 120 \AA$ for the monolayer deposited from solution as determined from the width of the dominant Bragg peak in the monolayer diffraction patterns. ${ }^{16,21}$ Annealing of the vapor-deposited monolayer does not increase its coherence length, indicating that the shorter-range translational order could be intrinsic to these films.

As noted in Ref. 16, neither monolayer structure is fully consistent with Groszek's model of molecular registry on the graphite (0001) surface. The intermolecular spacing in the vapor-deposited monolayer is too large for long-range commensurability with the graphite surface, whereas alternate molecules in the solution-deposited monolayer have their skeletal plane perpendicular to the surface.

In the present work, our aim is to elucidate the reason for the difference in the monolayer structure of these intermediate-length alkanes at room temperature depending on whether they are vapor deposited or deposited from solution. This effort led to investigating the monolayer structure of vapor-deposited films at somewhat higher coverage. Under these conditions, we expected to compress the alkane monolayer fully and thereby replicate the effect of the heptane solvent molecules adsorbing within the monolayer when depositing from solution. The sequence of phases revealed in these measurements led to a study of the monolayer structure below room temperature. Because of the similarity of the structures found for both the C24 and C32 films, we chose to concentrate our measurements on C24 monolayers. Except for the transition temperatures between phases, we do not believe the monolayer structures differ in any essential way.

\section{METHODS}

\section{A. Sample preparation}

Both the C24 and C32 films used in the present study were deposited onto a graphite substrate from the vapor phase. The sample preparation technique has been described in detail elsewhere. ${ }^{16,19,21,23,26}$ Briefly, the substrates were re- 
compressed exfoliated graphite (Grafoil), ${ }^{27}$ whose surface area was calibrated from a nitrogen vapor pressure adsorption isotherm at $77 \mathrm{~K}$. The $n-\mathrm{C}_{24} \mathrm{D}_{50}$ and $n-\mathrm{C}_{32} \mathrm{D}_{66}$ adsorbates were $98 \%$ deuterated and used as supplied. ${ }^{28}$ Appropriate charges of these alkanes were placed in a stainless steel cell containing the Grafoil substrate and sealed by a conflat flange fitted with a copper gasket. Vapor deposition of the films occurred upon heating the evacuated cell for $48 \mathrm{~h}$ to temperatures of $210{ }^{\circ} \mathrm{C}(\mathrm{C} 24)$ and $280{ }^{\circ} \mathrm{C}(\mathrm{C} 32)$.

\section{B. Neutron diffraction}

The use of completely deuterated alkane films greatly reduced the large incoherent neutron scattering from hydrogen. Our diffraction patterns from the polycrystalline monolayers on the highly uniform Grafoil substrates typically contain several Bragg peaks whose relative intensity is sensitive to the molecular orientation. In general, these peaks can be observed to a higher wave vector transfer $Q$ than is possible with $\mathrm{x}$-ray diffraction where the effect of the molecular form factor attenuates the scattering at high $Q .^{15,23,29-32}$

We conducted our neutron diffraction experiments on C24 and C32 monolayers on the two-axis diffractometer located at the C-port of the University of Missouri Research Reactor Facility. The neutron beam with wavelength of 4.35 $\AA$ scattered from the sample placed in a transmission geometry before reaching a five-detector data acquisition system. ${ }^{33}$ The temperature range investigated extended from 86 to 420 $\mathrm{K}$. It took approximately 3 days to collect a diffraction pattern at each temperature.

In order to correct for the background scattering coming from the Grafoil substrate, we collected diffraction patterns from the bare Grafoil at selected temperatures before the monolayers were deposited. The small temperature dependence of the Grafoil scattering did not warrant taking a background measurement at every temperature at which a film was investigated. To obtain the difference pattern from the monolayer film, we subtracted the diffraction pattern from the bare Grafoil measured at the temperature nearest to that of the vapor-deposited sample. Due to the large coherent cross section of our deuterated alkane films, we found it necessary to scale the bare Grafoil background scattering before subtraction in order to take into account the shielding of the Grafoil by the adsorbed film. The scale factor was selected to minimize the intensity in the difference pattern of both the graphite (002) Bragg peak and the intense smallangle scattering from the Grafoil particles. If the scale factor is too small, the graphite (002) peak intensity in the difference pattern will be large; on the other hand, if the scale factor is too large, the difference intensity becomes negative at low $Q$. In practice, we found that a scale factor of $\sim 0.85$ provided an acceptable intensity in the difference pattern for both the graphite small-angle scattering and its (002) peak.

To illustrate the background correction procedure, we show in Fig. 2(a) the diffraction pattern at $295 \mathrm{~K}$ from a 1.15-layer C24 film vapor deposited on a Grafoil substrate before subtraction of the bare-substrate background. The only scattering visible from the film is the small (11) Bragg peak at $Q=1.5 \AA^{-1}$. In Fig. 2(b), after subtracting the back-

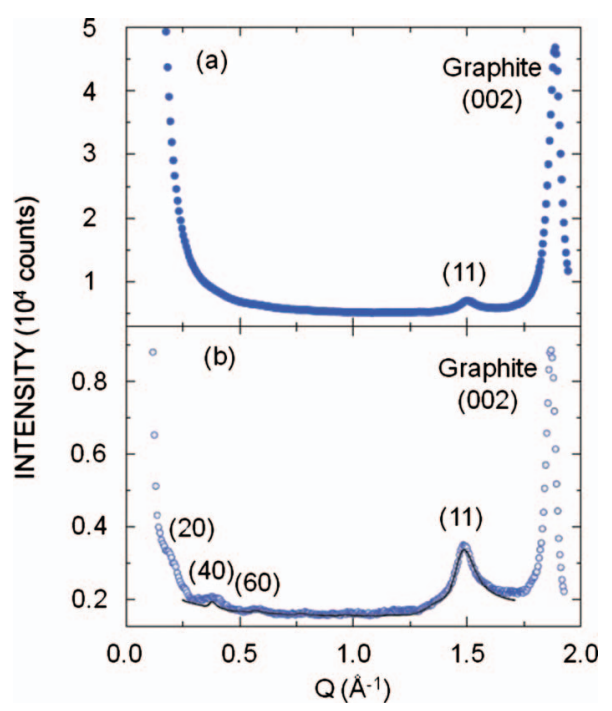

FIG. 2. Neutron diffraction pattern from a deuterated 1.15-layer $n-\mathrm{C}_{24} \mathrm{D}_{50}$ film at a temperature of $295 \mathrm{~K}$ : (a) before subtraction of the background scattering from the bare Grafoil substrate and (b) after background subtraction. In (b), several Bragg peaks in the $(h 0)$ series of reflections and the (11) peak from the $\mathrm{C} 24$ monolayer are visible. The solid line is the best fit to the data in the range of $0.25 \AA^{-1}<Q<1.65 \AA^{-1}$. The graphite (002) intensity is due to imperfect background subtraction.

ground, one sees three monolayer Bragg peaks in the $(h 0)$ series at low $Q$ as well as the (11) peak. Note that both the graphite (002) peak and the small-angle scattering from the Grafoil particles are still present but now at a level that allows analyzing the relative intensity of the monolayer Bragg peaks.

The background-corrected monolayer diffraction patterns were fit with a Warren lineshape ${ }^{34}$ using a refinement program for analyzing the structure of adsorbed polycrystalline films developed by one of us (K.W.H.). ${ }^{16,19}$ This profile analysis program obtains the best fit to the diffraction pattern by indexing it to a RC unit cell and varying the crystalline structural parameters as well as a linear background. The structure parameters include the $a$ and $b$ lattice constants, the coherence lengths $L_{a}$ and $L_{b}$ in these directions, and the rotation angles each molecule in the unit cell makes with three primary axes.

\section{MD simulations}

Details of our MD simulations have been described previously. ${ }^{20}$ We utilized a united atom model that replaces each methyl $\left(\mathrm{CH}_{3}\right)$ and methylene $\left(\mathrm{CH}_{2}\right)$ group by a pseudoatom of the same mass located at the group's carbon atom position. Bonds between pseudoatoms were constrained to a length of $1.53 \AA$, and molecular flexibility was modeled using angle-bend and dihedral-torsion potentials given by Weber. ${ }^{35}$ Intermolecular and molecule-substrate interactions were represented by Lennard-Jones 6-12 potentials. The simulations were conducted at constant number of molecules, volume, and temperature ( $N V T$ ensemble). They began with all molecules in their all-trans configuration and all bend angles at their equilibrium value of $109.0^{\circ}$. The simulation cell dimensions were selected to be commensurate with the graphite basal plane so that periodic boundary 
conditions could be applied. We adjusted the number of molecules and the size of the simulation cell to give a spreading pressure approximately equal to zero for the lower-density monolayer at $50 \mathrm{~K}$. For the higher-density monolayer, we used a larger spreading pressure resulting in a decrease in the monolayer $b$ lattice constant of $7 \%-8 \%$.

\section{RESULTS AND DISCUSSION}

In this section, we describe neutron diffraction measurements on vapor-deposited C24 and C32 films at two different coverages: slightly above monolayer completion and slightly below. At the higher coverage, we expected to compress the alkane monolayer fully, thereby replicating the effect of the heptane solvent molecules adsorbing within the monolayer when depositing from solution. In the case of the lower coverage, we wished to determine whether a transition to a denser phase might also occur upon cooling below room temperature.

\section{A. Phase transitions in a film at a coverage just above monolayer completion}

Figure 2(b) contains the diffraction pattern measured at room temperature from a 1.15-layer vapor-deposited C24 film where unity coverage is defined to be a complete layer having a rectangular unit cell with dimensions of $a=65 \AA$ and $b=4.53 \AA$ A. Recall that these cell dimensions correspond to a monolayer structure in which all molecules are oriented with their carbon skeletal plane parallel to the graphite surface, as shown in Fig. 1(b). Profile analysis of the pattern in Fig. 2(b) (solid curve) yielded a denser monolayer structure with lattice parameters of $a=66.0 \AA$ and $b=8.52 \AA$ $=2 \sqrt{3} a_{g}$, where $a_{g}=2.46 \AA$ is the graphite lattice constant. Here, we have assumed that the unit cell is doubled in the b-direction due to the alternating orientation of molecules within a lamella [see Fig. 1(d)]. The nearest-neighbor distance of molecules in this direction is $4.26 \AA$, which is in good agreement with the nearest-neighbor distance found in our previous neutron scattering measurements on both $\mathrm{C} 32$ and C24 monolayers deposited from a heptane solution onto graphite. ${ }^{16,19}$ It is also close to the value of $4.28 \AA$ found previously by x-ray diffraction from a $\mathrm{C} 32$ monolayer adsorbed from solution onto a graphite substrate. ${ }^{15}$

The intensity of the $(h 0)$ series of Bragg peaks is too weak in Fig. 2(b) to confirm the alternating orientation of the molecules from the profile analysis of the diffraction pattern as could be done for C24 and C32 monolayers deposited from solution. ${ }^{16,19}$ However, the agreement of the nearestneighbor distance of $4.26 \AA$ with that found for solutiondeposited monolayers, together with the evidence from MD simulations to be presented below, supports an alternating orientation of the molecules in the 1.15-layer vapordeposited C24 film.

Thus, we see that a small increment in coverage, just enough to initiate growth of a second layer, has the effect of uniaxially compressing the $\mathrm{C} 24$ monolayer into registry with the graphite basal surface. This behavior is the same as that observed for both C24 and C32 monolayers in the presence of a monolayer of $\mathrm{C} 7$ solvent. ${ }^{16,19}$ We suggest, then, that a
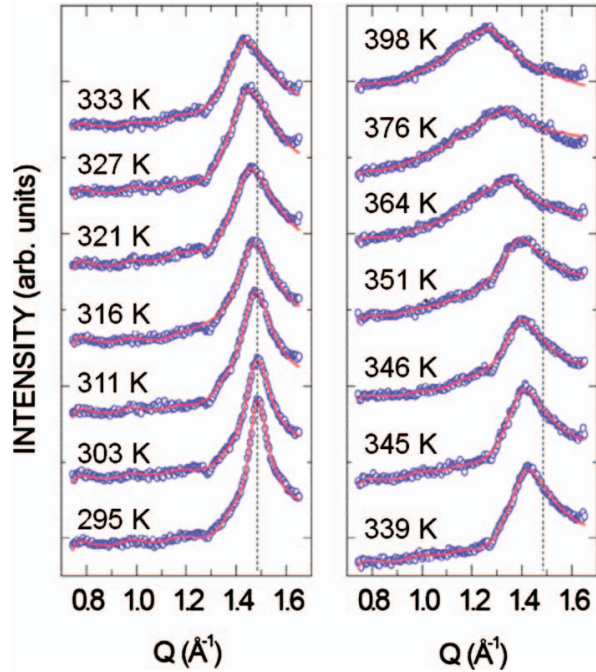

FIG. 3. Neutron diffraction patterns from the 1.15-layer C24 film adsorbed on Grafoil as a function of temperature. The open circles are data points and the solid curves are the best fit to a Warren line shape (Ref. 34). The vertical dashed line marks the position of the (11) Bragg peak of the fully compressed monolayer $(b=4.26 \AA)$. For each pattern, the background scan from the bare Grafoil taken at the temperature closest to that of the film has been subtracted. Note that the patterns are separated by a constant offset so that they do not superimpose.

small fraction of the solvent molecules enter the first layer of the film just as additional C24 molecules would and thereby increase the spreading pressure in the $\mathrm{C} 24$ layer. The compressive effect of the solvent forces the $\mathrm{C} 24$ layer into registry with the graphite surface in the $\mathbf{b}$-direction.

In our earlier study ${ }^{18}$ it was difficult to investigate the melting transition of the $\mathrm{C} 24$ and $\mathrm{C} 32$ monolayers in the presence of the $\mathrm{C} 7$ solvent molecules due to their desorption above room temperature. However, the low vapor pressure of the C24 film allows us to measure diffraction patterns from the 1.15-layer sample beginning in the compressed phase at room temperature [Fig. 1(d)] and upon heating up to its melting point. Figure 3 shows a series of diffraction patterns from the 1.15-layer film up to a temperature of $398 \mathrm{~K}$ in a range of wave vector transfers $Q$ near the monolayer (11) Bragg peak. The open circles are the data points, and the solid line is the best fit to a Warren line shape. ${ }^{34}$ As the temperature increases up to $311 \mathrm{~K}$, the (11) peak position remains fixed, indicating a nearly constant $d$-spacing, $d_{11}$, and $b$ lattice constant $(b$ $\approx d_{11}$ for $a \gg b$ ). However, we see the (11) peak width increases, corresponding to a decrease in the monolayer coherence length $L_{b}$ [note that with the large aspect ratio of the rectangular unit cell, the reciprocal lattice vector corresponding to the (11) Bragg reflection is nearly in the $\mathbf{b}$ direction]. Above $311 \mathrm{~K}$, thermal expansion begins with the (11) peak shifting to a lower $Q$ but with little change in $L_{b}$. The thermal expansion continues above $345 \mathrm{~K}$ along with a decrease in $L_{b}$.

The film's structural parameters upon heating can be viewed more easily in Fig. 4 where we have plotted (a) the $b$ lattice constant and (b) the coherence length $L_{b}$ as a function of temperature. While the onset of the film's thermal expansion is clearly seen at $\sim 310 \mathrm{~K}$ [Fig. 4(a)], the temperature dependence of $L_{b}$ is more revealing of the structural changes 


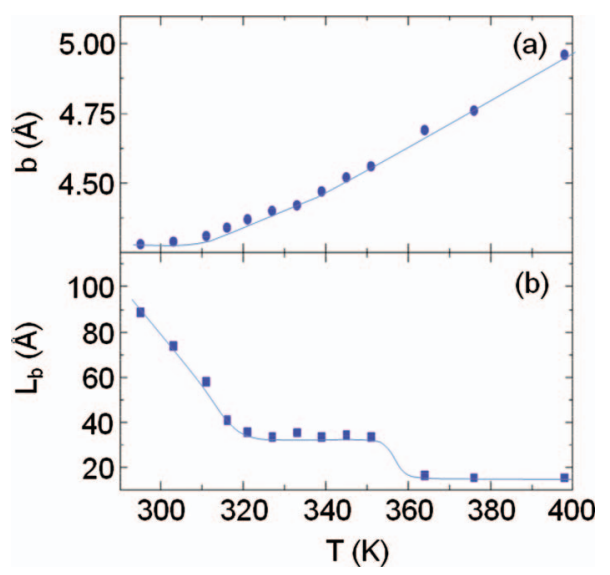

FIG. 4. (a) The $b$ lattice constant and (b) the coherence length in the b-direction $\left(L_{b}\right)$ as a function of temperature for the 1.15-layer C24 film adsorbed on Grafoil. These structural parameters are obtained from the fit to the diffraction patterns shown in Fig. 3. The solid curves are guides to the eye.

occurring in the film. As shown in Fig. 4(b), $L_{b}$ drops continuously from a value of $95 \AA$ at a temperature of $295 \mathrm{~K}$ to a value of $35 \AA$ at $320 \mathrm{~K}$ and then remains constant up to about $350 \mathrm{~K}$, where it drops to a value of $\sim 16 \AA$. This behavior of $L_{b}$ suggested that there could be three monolayer phases: (1) the crystalline phase that is uniaxially commensurate with the graphite surface at room temperature and which begins to expand on heating above $\sim 310 \mathrm{~K}$, (2) an incommensurate intermediate phase characterized by the plateau of $\sim 35 \AA$ in $L_{b}(320 \mathrm{~K}<T<350 \mathrm{~K})$, and (3) an isotropic fluid phase at high temperature $(T>360 \mathrm{~K})$.

In support of this interpretation, we note that the coherence length $L_{b} \sim 35 \AA$ and the range of $b$ lattice constants $(4.4-4.6 \AA)$ in the putative intermediate phase are close to those that we have found for vapor-deposited C24 and C32 monolayers at lower coverages and room temperature. ${ }^{16-18}$ Moreover, the drop in coherence length at $\sim 350 \mathrm{~K}$ in Fig. 4(b) is qualitatively similar to that observed at the melting transition for the lower-coverage vapor-deposited monolayers [see Fig. 2(b) in Ref. 18].

It is interesting to compare our neutron diffraction results with a study by Askadskaya and Rabe using STM of a C24 monolayer adsorbed on graphite from a phenyloctane solution. ${ }^{14}$ They found a lattice constant of $b=4.25 \AA$ at room temperature close to the value of $4.26 \AA$ for our 1.15 layer C24 film as well as for our C24 and C32 monolayers deposited from solution. Upon heating, they observed the lamella boundaries to broaden continuously until at $\sim 318 \mathrm{~K}$ they could not resolve any molecular order. Their apparent transition temperature near $318 \mathrm{~K}$ is close to our temperature of $320 \mathrm{~K}$ at which we see the coherence length level off in the intermediate phase.

In addition to these STM measurements,${ }^{14}$ both our MD simulations to be discussed below and our quasielastic neutron scattering experiments ${ }^{5,6}$ indicate some translational diffusive motion within the lamellas of C24 monolayers below the melting transition. Therefore, we are motivated to identify the intermediate phase of the 1.15-layer C24 film with a "smectic" phase of shorter-range translational order than the

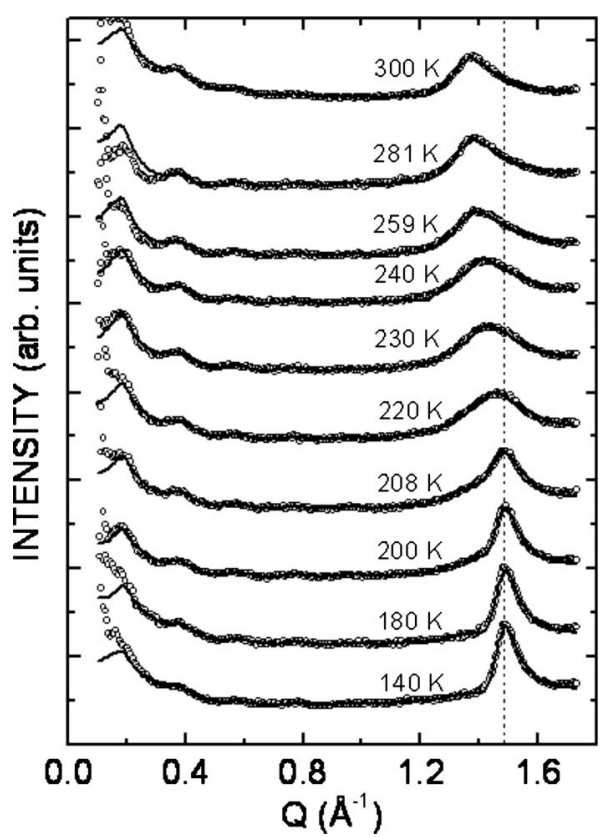

FIG. 5. Neutron diffraction patterns from a 0.94-layer $n-\mathrm{C}_{24} \mathrm{D}_{50}$ film adsorbed on Grafoil as a function of temperature for $T \leq 300 \mathrm{~K}$. The open circles represent data points, while the solid line is the best-fit profile calculated assuming a RC cell as shown in Fig. 1(d) for $T \leq 208 \mathrm{~K}$ and Fig. 1 (b) for $220 \mathrm{~K} \leq T \leq 300 \mathrm{~K}$. The vertical dashed line marks the position of the (11) Bragg peak of the fully compressed monolayer $(b=4.28 \AA$ ).

low-temperature crystalline phase and characterized by broadened lamellar boundaries. We note that our use of the term smectic differs somewhat from that of Askadskaya and Rabe $^{14}$ who apply it to an apparently metastable monolayer phase of $\mathrm{C} 24$ on graphite at $329 \mathrm{~K}$. The molecular mobility in this phase is too high to achieve molecular resolution in the STM images; but they were able, nevertheless, to observe lamellas with the same width and orientation as the lowtemperature crystalline phase. Surprisingly, they found that the lamella boundaries in this smectic phase are not broadened as in the smectic phase that we have identified in our MD simulations.

\section{B. Phase transitions in films at a coverage just below monolayer completion}

The evidence of three different monolayer phases of the 1.15-layer C24 film presented in Sec. III A suggested that a lower-coverage C24 or C32 film might also exhibit three phases but in a lower temperature range. In particular, a crystalline phase characterized by a larger coherence length might occur at a lower temperature than previously investigated. We therefore began experiments below room temperature on lower-coverage films.

We show diffraction patterns from a 0.94-layer vapordeposited C24 film in the range of 140-300 K in Fig. 5. As anticipated, we see that the behavior upon heating is qualitatively similar to that of the 1.15-layer C24 film in Fig. 3. The position and width of the (11) peak do not change significantly up to a temperature of $\sim 208 \mathrm{~K}$, at which point the peak begins to shift gradually to a lower $Q$ and broaden abruptly. 


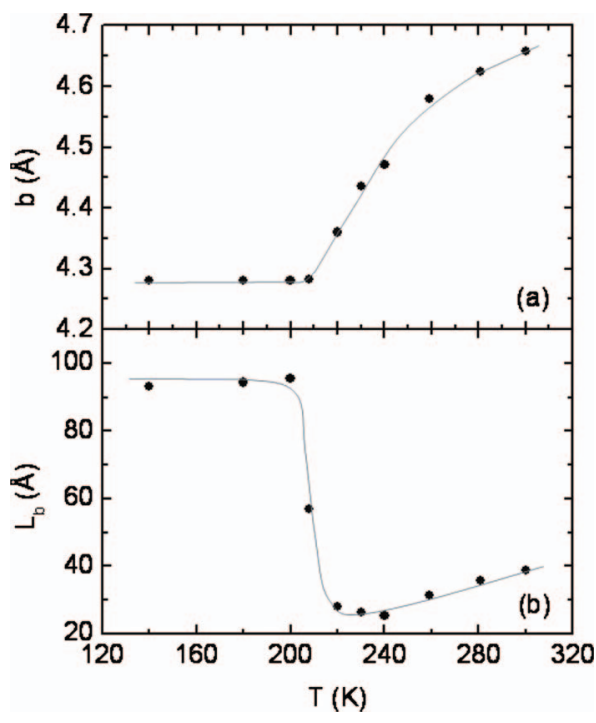

FIG. 6. (a) The $b$ lattice constant and (b) the coherence length $L_{b}$ for the 0.94-layer C24 film as a function of temperature. These structural parameters are obtained from fitting the diffraction patterns shown in Fig. 5. The solid curves are guides to the eye.

In Fig. 6, we have plotted the temperature dependence of the $b$ lattice constant and coherence length $L_{b}$ for the 0.94layer $\mathrm{C} 24$ film obtained from the best fit to the diffraction patterns in Fig. 5. One sees that $b$ remains constant with a value of $4.28 \AA$ at temperatures up to $\sim 208 \mathrm{~K}$ and then gradually increases upon heating up to room temperature. Similarly, $L_{b}$ remains constant with a value of $\sim 95 \AA$ until it abruptly decreases near $208 \mathrm{~K}$. On comparing these results with those of the 1.15-layer C24 film in Fig. 4, we interpret this behavior as indicating that the 0.94-layer C24 film undergoes a crystalline-to-smectic phase transition in the temperature range of $208-220 \mathrm{~K}$, which is about $100 \mathrm{~K}$ below the corresponding transition temperature for the 1.15-layer film.

We attribute the small upturn in $L_{b}$ above $240 \mathrm{~K}$ in Fig. 6(b) to an artifact of our fitting procedure. There is a weak shoulder on the low- $Q$ side of the (11) Bragg peak as shown in Fig. 7 that reaches its greatest intensity at $\sim 200 \mathrm{~K}$. In our fitting procedure, we introduced a Gaussian component to represent this shoulder. We suggest that it is produced by a small fraction of $\mathrm{C} 24$ in the lower-density smectic phase that coexists with the crystalline phase close to the crystalline-tosmectic transition. However, in the temperature range of 220-240 K, this shoulder could not be resolved so that intensity from both coexisting phases was included in the fit of the (11) peak. This produced an artificially broadened line shape and resulted in an underestimate of the coherence length in the range of $220-240 \mathrm{~K}$.

The quality of these diffraction patterns permits a more detailed structural analysis than possible for the 1.15-layer film in Fig. 2(b). As an example, we show in Fig. 7 the diffraction pattern from the 0.94-layer C24 film at a temperature of $208 \mathrm{~K}$. We see that the $(h 0)$ series of Bragg reflections is well resolved and is fitted well (solid curve) by the model in Fig. 1(d) in which the carbon skeletal planes of neighboring molecules alternate between an orientation parallel and perpendicular to the graphite surface within a

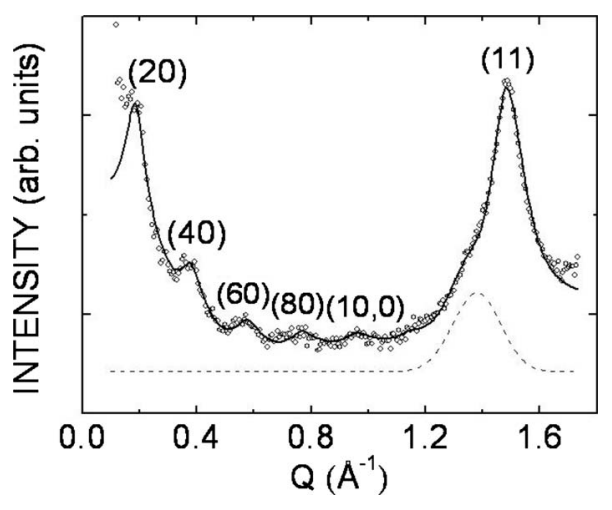

FIG. 7. The diffraction pattern from the 0.94-layer C24 film measured at a temperature of $208 \mathrm{~K}$ (same pattern as shown in Fig. 5). The background from bare Grafoil has been subtracted. The circles represent the data points, while the solid line is the best fit to a model in which the carbon skeletal planes alternate between parallel and perpendicular within a lamella [see Fig. 1(d)]. The structural parameters for this best fit are $a=66.3 \AA$, $b=4.28 \AA, L_{a}=69 \AA$, and $L_{b}=57 \AA$. The dashed line corresponds to a Gaussian line shape that has been added to the model in order to fit the shape of the leading edge of the (11) peak. This Gaussian component may be due to coexistence of the low-density phase (see text).

lamella. An all-parallel orientation of molecules as in Fig. 1(b) did not reproduce as well the intensity of the $(h 0)$ series of peaks relative to that of the dominant (11) peak. The bestfit parameters of the rectangular unit cell are $a=66.3 \AA$ and $b=4.28 \AA$. Due to the proximity to the crystalline-to-smectic phase transition, the coherence length $L_{b}=57 \AA$ is somewhat smaller than its value of $93 \AA$ at the lowest temperature of $140 \mathrm{~K}$. This structure is in reasonable agreement with that found at room temperature for C24 and C32 monolayers deposited from solution ${ }^{16,17,19}$ and for our 1.15-layer C24 film.

We have found evidence that a submonolayer of the somewhat longer alkane, C32, vapor deposited onto a Grafoil substrate also undergoes a smectic-to-crystalline phase transition upon cooling below room temperature. In Fig. 8, we see that the diffraction pattern at $86 \mathrm{~K}$ shows a shift in the position of the (11) peak to higher $Q$ and a decrease in the width of its leading edge compared to the pattern at room temperature. These features are consistent with a transition upon cooling to a uniaxially commensurate monolayer $(b=4.26 \AA)$ with a longer coherence length $(\sim 100 \AA)$.

Previous studies have investigated the melting transition of monolayer C24 and C32 films on graphite, which, with our identification of the smectic phase, can now be termed the smectic-to-fluid transition. ${ }^{17,19}$ In Fig. 9, we show the diffraction patterns above room temperature from vapordeposited films of (a) 1.00-layer C32 and (b) 0.97-layer C24 from Ref. 17. We find good agreement with the roomtemperature diffraction patterns shown previously for C24 and C32 monolayers in Figs. 5 and 8, respectively; the position of the (11) peak of both films is consistent with a smectic phase $\left(d_{11} \approx 4.6 \AA\right)$. As was true for the smectic phase of the 1.15-layer $\mathrm{C} 24$ film, the peak position and width are relatively insensitive to temperature up to the melting points of $350 \mathrm{~K}(\mathrm{C} 32)$ and $340 \mathrm{~K}(\mathrm{C} 24)$ where they broaden abruptly and shift gradually to lower $Q$.

In Fig. 10, we summarize in a one-dimensional diagram 


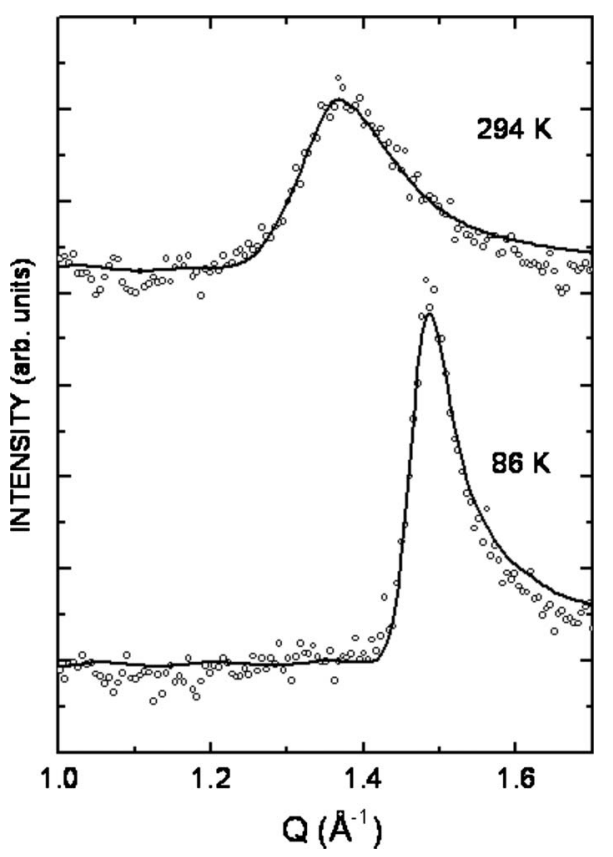

FIG. 8. Neutron diffraction patterns at two different temperatures from a 1.03-layer C32 film vapor-deposited onto a Grafoil substrate. At $86 \mathrm{~K}$, the film is in a uniaxial commensurate phase similar to that shown in Fig. 1(d), while at $294 \mathrm{~K}$ the film has transformed to the incommensurate smectic phase [Fig. 1(b)]. The increased scatter in the data points (cf. Fig. 7) is due to the small size of the sample, which was fabricated for quasielastic neutron scattering measurements.

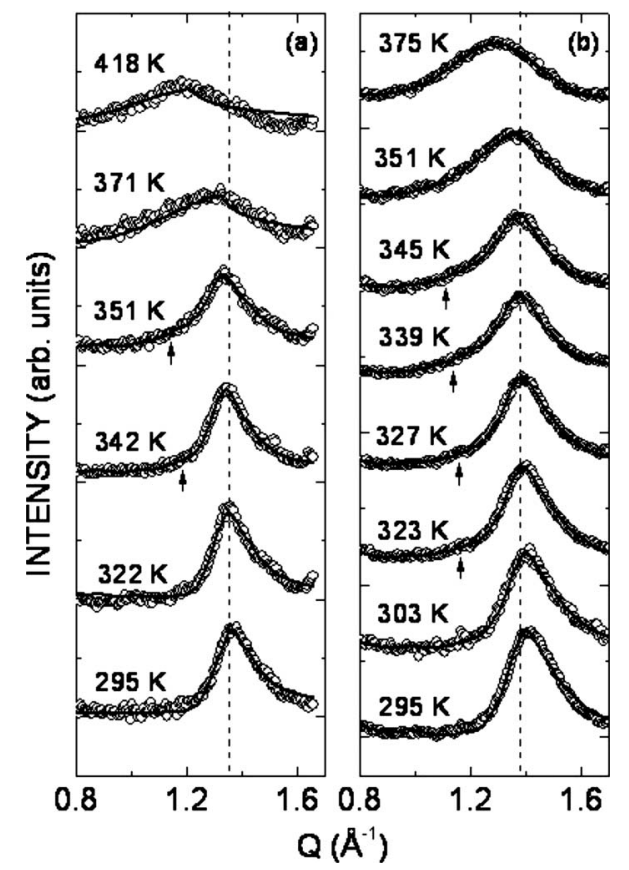

FIG. 9. Temperature dependence of the neutron diffraction patterns from vapor-deposited samples for $T \geq 295 \mathrm{~K}$ : (a) a 1.00-layer $n-\mathrm{C}_{32} \mathrm{D}_{66}$ film and (b) a 0.97-layer $n-\mathrm{C}_{24} \mathrm{D}_{50}$ film (from Ref. 17). The solid curves are fits to the diffraction patterns using the model in Fig. 1(b) in which the skeletal plane of all the molecules is oriented parallel to the graphite surface. The dashed vertical lines indicate the position of the monolayer (11) peak at room temperature $(b=4.56 \AA)$. The arrows indicate the location of weak Gaussian components that were included in the solid curve to improve the fit to the diffraction patterns (see text). (a)

$$
0.94 \text { layer }
$$

$\underset{\sim 215}{\stackrel{\text { crystalline }}{2} \text { smectic }} \stackrel{\text { fluid }}{\longrightarrow} \underset{340}{\longrightarrow}$

(b)

1.15 layer

\begin{tabular}{|c|c|c|}
\hline crystalline & \multicolumn{2}{|c|}{ smectic fluic } \\
\hline & 320 & 355 \\
\hline
\end{tabular}

FIG. 10. One-dimensional phase diagrams comparing the crystalline-tosmectic and smectic-to-fluid phase transition temperatures inferred from the temperature dependence of the coherence length for (a) the 0.94-layer C24 film in Fig. 6(b) and (b) the 1.15-layer C24 film in Fig. 4(b).

the phase transition temperatures inferred for (a) the lowercoverage (0.94-layer) and (b) higher-coverage (1.15-layer) C24 films. As discussed above, the higher-coverage film exhibits all three monolayer phases, crystalline, smectic, and fluid above room temperature, whereas the lower-coverage film is already in the smectic phase at $300 \mathrm{~K}$. We see that the crystalline-to-smectic phase transition temperature is very sensitive to coverage, occurring at a temperature about 100 $\mathrm{K}$ lower in the submonolayer film compared to the fully compressed monolayer (1.15 layers).

\section{Comparison with MD simulations}

Figure 11 shows "snapshots" from C24 monolayer simulations at three different temperatures. Each snapshot is a top view of the simulation cell containing 32 C24 molecules adsorbed on a graphite basal-plane surface and arranged in four lamellas. The panels on the left correspond to a lowdensity C24 monolayer phase for which the spreading pressure is approximately zero at a temperature of $50 \mathrm{~K}$; it is intended to simulate a submonolayer C24 film. The panels on the right correspond to a higher-density phase obtained by increasing the spreading pressure.

These simulations provide some insight into the sequence of phase transitions that we have observed for C24 (a)

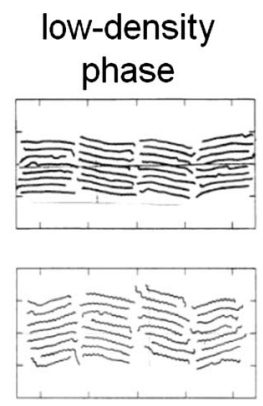

(c)

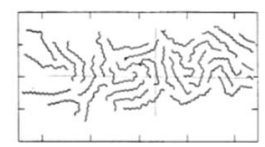

high-density phase
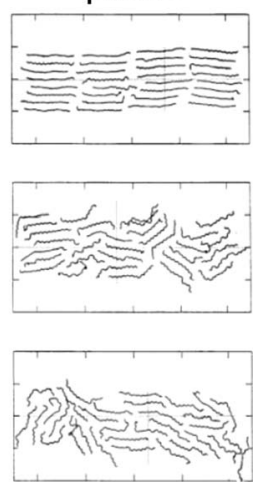

FIG. 11. Snapshots showing top view ( $x-y$ plane) of the MD simulation cell for a low-density (left) and high-density (right) C24 monolayer at three different temperatures: (a) $300 \mathrm{~K}$, (b) $325 \mathrm{~K}$, and (c) $350 \mathrm{~K}$. 

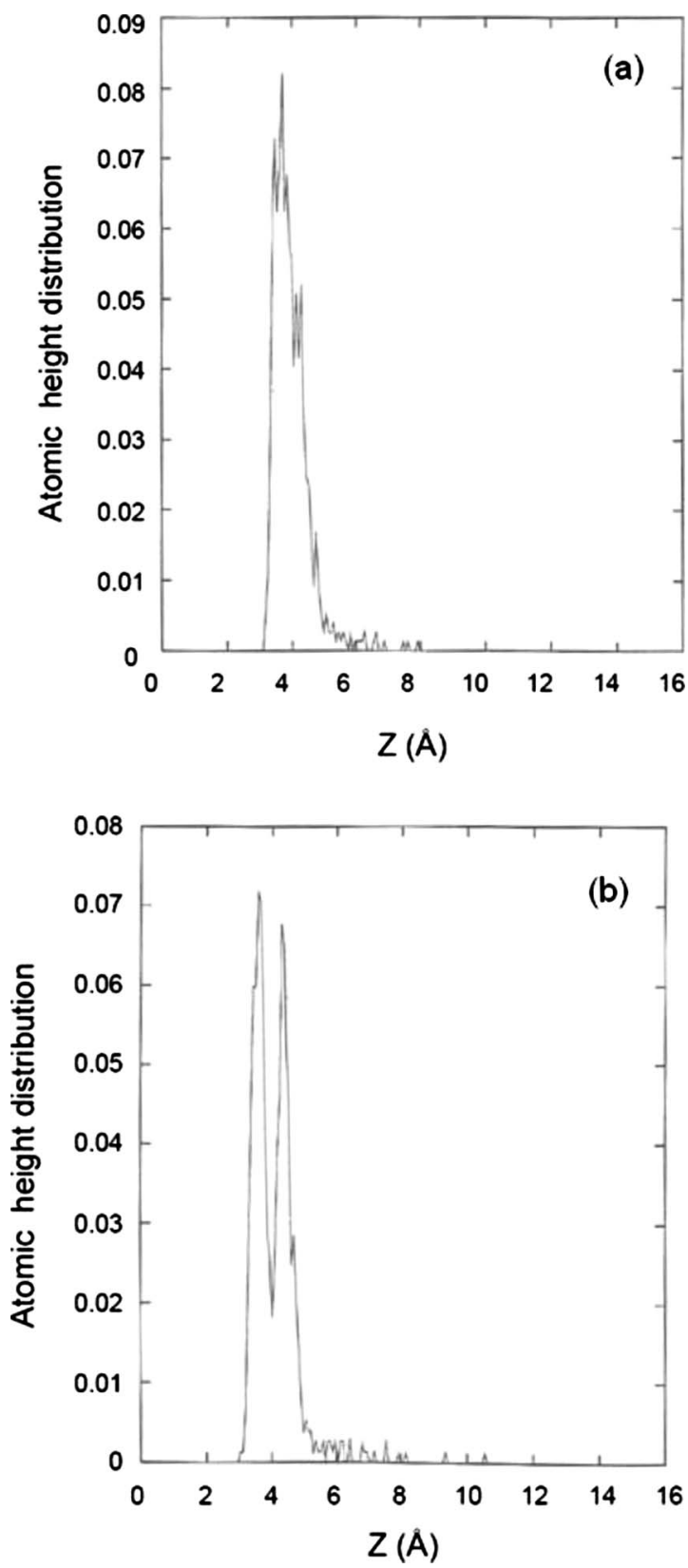

FIG. 12. Distribution of pseudoatom distances from the surface calculated from the simulation of (a) the low-density $\mathrm{C} 24$ monolayer at a temperature of $325 \mathrm{~K}$ and (b) the high-density C24 monolayer at $300 \mathrm{~K}$.

and C32 monolayers adsorbed on graphite. At a temperature of $300 \mathrm{~K}$, the simulation snapshots for both $\mathrm{C} 24$ monolayer densities show relatively well-ordered lamellas [see Fig. 11(a)]. Most of the molecules remain in the all-trans configuration with only a few gauche defects (kinks) located predominantly at the chain ends. At the lower density, most of the molecules are oriented with their carbon skeletal plane parallel to the graphite surface so that in a top view one sees the zigzag shape of their carbon backbone. However, at the higher density some of the molecules have rotated $90^{\circ}$ about their long axis so that the zigzag shape is no longer visible. These molecular reorientations are substantiated in Figs. 12(a) and 12(b), which show the distribution of the pseudoatom distances from the graphite surface for the low-density and high-density simulations, respectively. In the high- density simulation, there is clearly a double peak in the height distribution, indicating about the same number of C24 molecules with their carbon skeletal plane perpendicular to the surface as parallel. Thus, the high-density simulation is consistent with a monolayer structure in which the molecules alternate in orientation, as shown in Fig. 1(d). We propose this relatively well-ordered higher-density crystalline structure for the 1.15-layer $\mathrm{C} 24$ film below $318 \mathrm{~K}$ and for the 0.97-layer film below $\sim 200 \mathrm{~K}$, where, in both cases, the monolayer coherence length exceeds $35 \AA$.

At a higher temperature of $325 \mathrm{~K}$, the snapshots of the simulation cells in Fig. 11(b) show the lamella boundaries beginning to meander in the lower-density simulation and the lamellar structure virtually disappearing at higher density. The greater disorder at the higher density may result from gauche defect creation to relieve the larger spreading pressure. At both densities, the molecules contain a large number of gauche defects still largely confined to the chain ends. There is also a noticeable increase in the intermolecular separation within the lamellas from that at lower temperature. We associate these more poorly ordered lamellar structures with the smectic phase where we observe the coherence length to level off at $\sim 35 \AA$ [see Figs. 4(b) and 6(b)]. For the $\mathrm{C} 24$ films, the smectic phase occurs in the temperature range from 318 to $350 \mathrm{~K}$ (1.15 layers) and from 220 to 340 $\mathrm{K}$ (0.97 layer) as diagrammed in Fig. 10. Again, we note the substantially wider temperature range of the smectic phase at the submonolayer coverage.

At still higher temperature, the lamellar structure of the C24 monolayer disappears at both densities as shown in Fig. $11(\mathrm{c})$ at $350 \mathrm{~K}$. We identify this change with a transition to a fluid phase. The molecules now have a more globular shape due to the presence of gauche defects in the central region of the molecular chains. The fluid phase is characterized in the diffraction patterns of the $\mathrm{C} 24$ films by a decrease in coherence length below $\sim 15 \AA$, which is observed to occur above $350 \mathrm{~K}$ at a coverage of 1.15 layers and above $340 \mathrm{~K}$ at a coverage of 0.97 layer (see Fig. 10).

The MD simulations also give some insight into the type of diffusive motion occurring in the smectic phase. From following trajectories of the molecules in the simulation snapshots [not shown in Fig. 11(b)], one finds that the motion of the C24 molecules within a lamella is highly correlated. In particular, there is a tendency of the molecules to translate parallel to their long axis. Evidence of this diffusive motion can also be seen in the center-of-mass velocity autocorrelation functions, $V_{x}, V_{y}$, and $V_{z}$, for motion of the molecules in the $x, y$, and $z$ directions, respectively, as calculated from the simulations. In Fig. 13, we show the velocity correlation functions of a low-density C32 monolayer at $300 \mathrm{~K}$, which we identify as being in the smectic phase. In the $z$-direction normal to the surface, the molecules perform a bouncing motion as indicated by the damped oscillatory behavior of $V_{z}$. Similarly, in the $y$-direction, the molecules move toward and away from each other so that $V_{y}$ also has oscillatory behavior but one that is more strongly damped than in the $z$-direction. In contrast, in the $x$-direction that is 


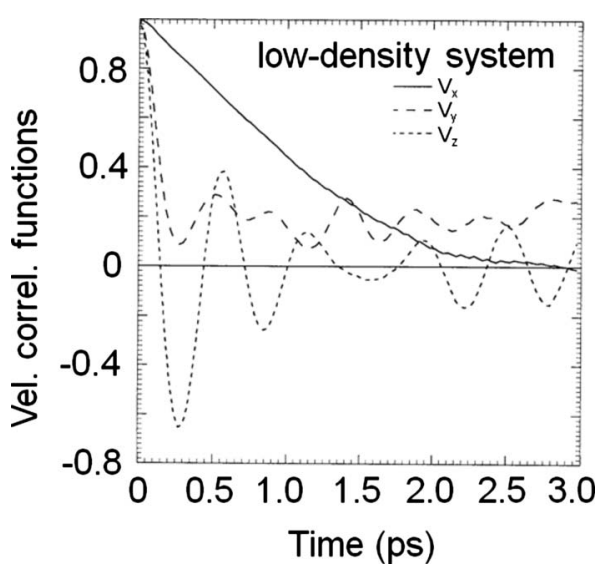

FIG. 13. Center-of-mass velocity correlation functions, $V_{x}, V_{y}$, and $V_{z}$ calculated from the MD simulations of a low-density C 32 monolayer. The $x$-direction is parallel to the long axis of the molecules, and the $z$-direction is normal to the graphite surface. No quantitative uncertainty analysis was performed, but the calculated data points are based on simulations with a standard deviation of the total energy on the $1 \%$ level.

nearly parallel to the long axis of the molecule, $V_{x}$ in Fig. 13, has an exponential decay indicating the molecular motion is diffusive in character.

The presence of highly correlated motion in the smectic phase found in the MD simulations also provides an explanation of why we observe a diffraction peak above $\sim 318 \mathrm{~K}$ of the 1.15-layer C24 film even though STM images in a similar film showed no translational order at these temperatures. ${ }^{14}$ Longitudinal diffusive motion parallel to the long molecular axis preserves the nearest-neighbor spacing of the molecules within a lamella well enough for the (11) Bragg peak to be observable in a diffraction experiment, although it is broader and weaker than in the crystalline phase. On the other hand, due to its slow scan speed ( $\sim 1 \mathrm{~ms} /$ line), STM cannot distinguish between the smectic phase in which there is correlated motion within a lamella and a fluid phase.

While the MD simulations are helpful in suggesting the sequence of C24 and C32 monolayer phases observed upon heating as well as reconciling the neutron diffraction and STM measurements, there are some limitations in using them to interpret our neutron diffraction measurements at this point. The simulations of the low-density C24 monolayer in a constant $N V T$ ensemble do not quantitatively reproduce the crystalline-to-smectic phase transition temperature. Also, it is unclear whether the greater spreading pressure used in the higher-density simulation actually reproduces the effect of a partial second layer present in the case of the 1.15-layer C24 film. Although we performed a simulation on a 1.15-layer film (i.e., by increasing the number of molecules in the lowdensity simulation by $15 \%$ ), the additional molecules did not enter the first layer to form a high-density monolayer upon equilibration at low temperature. In addition, the highdensity monolayer simulations did not yield a structure with a unit cell that was uniaxially commensurate with the graphite surface as inferred from our diffraction patterns. These results raise the question of whether the united atom model of the C24 molecule used in the simulations is adequate to sense the corrugation of the graphite substrate and thereby reproduce the smectic-to-crystalline transition upon cooling.

Very recent all-atom MD simulations of a C24 monolayer do reveal evidence of a transition to an intermediate phase near $250 \mathrm{~K}^{36}$ At this point, it is not clear whether this transition results from explicitly including the hydrogen atoms in the C24 molecules or whether it would occur in a united atom model if the same order parameters (measures of four and six nearest neighbors, respectively) were used in analyzing the simulation. It is also not clear why these order parameters in the all-atom simulations show a change back toward their low-temperature values upon heating through the melting point.

\section{SUMMARY AND CONCLUSION}

Our neutron diffraction measurements on a C24 film having a coverage slightly above monolayer completion show the same structure as that found for a C24 submonolayer deposited from a solution with heptane. We attribute this similarity to an increase in spreading pressure caused by the solvent molecules entering the first layer of the film.

For C24 and C32 monolayers vapor deposited on graphite basal-plane surfaces, we find evidence of three different phases: a crystalline phase at low temperature that transforms upon heating to an intermediate or smectic phase, followed by a melting transition to an isotropic fluid phase. The lowtemperature crystalline phase has the same lamellar structure as that of a submonolayer deposited from solution at room temperature. Within a lamella, the molecules alternate between an orientation in which the carbon skeletal plane is parallel to the surface and perpendicular to it, as shown in Fig. 1(d). The smectic phase preserves the lamellar structure of the crystalline phase; however, there is diffusive motion of the molecules within the lamellas. In particular, the lamella boundaries are broadened due to sliding of the molecules in a direction parallel to their long axis. The temperature range over which the smectic phase is stable depends strongly on coverage near monolayer completion.

We speculate that the much lower crystalline-to-smectic phase transition temperature in the 0.94-layer sample (see Fig. 10) may result from nucleation of the smectic phase at the boundaries of submonolayer islands where the molecules have fewer nearest neighbors. This feature is not reproduced in the simulations because the low-density phase is modeled as a homogenous medium filling the simulation cell (see Fig. 11). However, in the 1.15-layer sample, the expansion in the b-direction required for the transition from the crystalline to the smectic phase necessitates promotion of some of the molecules or parts of them to the second layer. This layer promotion could broaden or delay the transition on heating. Again, the simulations could not corroborate this behavior. As noted above, when the number of molecules in the lowdensity simulation was increased by $15 \%$, the additional molecules did not enter the first layer to form a high-density phase upon equilibration at low temperature.

To summarize, our present results demonstrate two routes to reaching the crystalline monolayer phases of C24 and C32 with alternating molecular orientations. Beginning 
in the smectic phase at room temperature, one can transform the monolayer to this crystalline phase either by cooling the film or by compressing it through increasing coverage. Our previous results ${ }^{16}$ have shown that the necessary compression can also be achieved through the addition of solvent molecules.

We conclude by suggesting that the smectic phase mediating the crystalline and fluid phases of these monolayers of intermediate-length alkanes may be considered as the twodimensional (2D) analog of the solid rotator phases that precede the melting of three-dimensional alkane crystals. ${ }^{37}$ In particular, nanoparticles of C32 supported on a solid surface are believed to undergo a transition from an orthorhombic structure at low temperature to a nearly hexagonal phase in which the molecules diffuse rotationally about their long axis and translate parallel to it. ${ }^{3,38}$ Quasielastic neutron scattering measurements and MD simulations are consistent with both of these types of molecular diffusive motion occurring in the C24 monolayer smectic phase along with motions associated with conformational changes in the molecules. ${ }^{4}$ The neutron diffraction and MD results that we have presented here indicate that these diffusive motions occur within a 2D lamellar structure characterized by a shorter coherence length than the low-temperature crystalline phase.

\section{ACKNOWLEDGMENTS}

The authors acknowledge helpful discussions with $\mathrm{B}$. Kuchta and L. Firlej. This work was supported by U.S. National Science Foundation under Grant Nos. DMR-0411748 and DMR-0705974.

${ }^{1}$ T. K. Xia and U. Landman, Science 261, 1310 (1993).

${ }^{2}$ K. R. Paserba and A. J. Gellman, Phys. Rev. Lett. 86, 4338 (2001).

${ }^{3}$ M. Bai, K. Knorr, M. J. Simpson, S. Trogisch, H. Taub, S. N. Ehrlich, H. Mo, U. G. Volkmann, and F. Y. Hansen, Europhys. Lett. 79, 26003 (2007).

${ }^{4}$ A. D. Enevoldsen, F. Y. Hansen, A. Diama, H. Taub, R. M. Dimeo, D. A Neumann, and J. R. D. Copley, J. Chem. Phys. 126, 104704 (2007).

${ }^{5}$ F. Y. Hansen, L. Criswell, D. Fuhrmann, K. W. Herwig, A. Diama, R. M. Dimeo, and D. A. Neumann, Phys. Rev. Lett. 92, 046103 (2004).

${ }^{6}$ H. Taub, F. Y. Hansen, L. Criswell, D. Fuhrmann, K. W. Herwig, A. Diama, H. Mo, R. M. Dimeo, D. A. Neumann, and U. G. Volkmann, AIP Conf. Proc. 708, 201 (2004).

${ }^{7}$ A. J. Groszek, Proc. R. Soc. London, Ser. A 314, 473 (1970).

${ }^{8}$ H. E. Kern, A. Piechocki, U. Brauer, and G. H. Findenegg, Prog. Colloid
Polym. Sci. 65, 118 (1978).

${ }^{9}$ G. H. Findenegg and M. Liphard, Carbon 25, 119 (1987).

${ }^{10}$ G. C. Mcgonigal, R. H. Bernhardt, and D. J. Thomson, Appl. Phys. Lett. 57, 28 (1990).

${ }^{11}$ G. Watel, F. Thibaudau, and J. Cousty, Surf. Sci. Lett. 281, L297 (1993).

${ }^{12}$ J. P. Rabe and S. Bucholz, Phys. Rev. Lett. 66, 2096 (1991).

${ }^{13}$ J. P. Rabe and S. Bucholz, Science 253, 424 (1991).

${ }^{14}$ L. Askadskaya and J. P. Rabe, Phys. Rev. Lett. 69, 1395 (1992).

${ }^{15}$ K. Morishige, Y. Takami, and Y. Yakota, Phys. Rev. B 48, 8277 (1993).

${ }^{16}$ K. W. Herwig, B. Matthies, and H. Taub, Phys. Rev. Lett. 75, 3154 (1995).

${ }^{17}$ H. Taub, K. W. Herwig, B. Matthies, and F. Y. Hansen, Inorg. Mater. 35, 847 (1999).

${ }^{18}$ F. Y. Hansen, K. W. Herwig, B. Matthies, and H. Taub, Phys. Rev. Lett. 83, 2362 (1999).

${ }^{19}$ B. Matthies, Ph.D. thesis, University of Missouri (1999).

${ }^{20}$ A. D. Enevoldsen, F. Y. Hansen, A. Diama, L. Criswell, and H. Taub, J. Chem. Phys. 126, 104703 (2007).

${ }^{21}$ L. Criswell, Ph.D. thesis, University of Missouri (2007).

${ }^{22}$ H. Mo, S. Trogisch, H. Taub, S. N. Ehrlich, U. G. Volkmann, F. Y. Hansen, and M. Pino, Phys. Status Solidi A 201, 2375 (2004).

${ }^{23}$ H. Mo, Ph.D. thesis, University of Missouri (2004).

${ }^{24}$ U. G. Volkmann, M. Pino, L. A. Altamirano, H. Taub, and F. Y. Hansen, J. Chem. Phys. 116, 2107 (2002).

${ }^{25}$ H. Mo, H. Taub, U. G. Volkmann, M. Pino, S. N. Ehrlich, F. Y. Hansen, E. Lu, and P. Miceli, Chem. Phys. Lett. 377, 99 (2003).

${ }^{26} \mathrm{~K}$. W. Herwig, B. Matthies, and H. Taub, in Neutron Scattering in Materials Science, MRS Symposia Proceedings No. 376, edited by D. A. Neumann, T. P. Russell, and B. J. Wuensch (Materials Research Society, Pittsburgh, 1994), p. 757.

${ }^{27}$ UCAR Carbon Co., P.O. Box 94637, Cleveland, OH 44101.

${ }^{28}$ Cambridge Isotope Laboratories, 50 Frontage Road, Andover, MA 01810.

${ }^{29}$ H. Taub, in Time Domain in Surface and Structural Dynamics, NATO Advanced Studies Institute, Series C: Mathematical and Physical Sciences, Vol. 228, edited by G. J. Long and F. Grandjean (Kluwer, Dordrecht, 1988), p. 467.

${ }^{30}$ T. Arnold, R. K. Thomas, M. A. Castro, S. M. Clarke, L. Messe, and A. Inaba, Phys. Chem. Chem. Phys. 4, 345 (2002).

${ }^{31}$ T. Arnold, C. C. Dong, R. K. Thomas, M. A. Castro, A. Perdigon, S. M. Clarke, and A. Inaba, Phys. Chem. Chem. Phys. 4, 3420 (2002).

${ }^{32}$ Z. Wu, S. N. Ehrlich, B. Matthies, K. W. Herwig, P. Dai, U. G. Volkmann, F. Y. Hansen, and H. Taub, Chem. Phys. Lett. 348, 168 (2001).

${ }^{33}$ R. Berliner, D. F. Mildner, J. Sudol, and H. Taub, in Position Sensitive Detection of Thermal Neutrons, edited by P. Convert and J. B. Forsythe (Academic, New York, 1983), p. 120.

${ }^{34}$ B. E. Warren, Phys. Rev. 59, 693 (1941).

${ }^{35}$ T. A. Weber, J. Chem. Phys. 69, 2347 (1978).

${ }^{36}$ L. Firlej, B. Kuchta, M. W. Roth, M. J. Connolly, and C. Wexler, Langmuir 24, 12392 (2008).

${ }^{37}$ E. B. Sirota, H. E. King, Jr., D. M. Singer, and H. H. Shao, J. Chem. Phys. 98, 5809 (1993).

${ }^{38}$ H. G. Olf and A. Peterlin, J. Polym. Sci., Part A-2 8, 791 (1970). 